\title{
microRNA-222 promotes tumor growth and confers radioresistance in nasopharyngeal carcinoma by targeting PTEN
}

\author{
WEI WU ${ }^{1}$, XI CHEN ${ }^{1}$, SHILONG YU ${ }^{2}$, RUI WANG ${ }^{3}$, RUIKUN ZHAO ${ }^{1}$ and CHAO DU $^{4}$ \\ Departments of ${ }^{1}$ Radiation Oncology and ${ }^{2}$ Intervention, Tumor Hospital of Jilin Province, Changchun, Jilin 130012; \\ Departments of ${ }^{3}$ Radiation and ${ }^{4}$ Neurosurgery, China-Japan Union Hospital of Jilin University, \\ Changchun, Jilin 130033, P.R. China
}

Received August 31, 2016; Accepted October 10, 2017

DOI: $10.3892 / \mathrm{mmr} .2017 .7931$

\begin{abstract}
MicroRNA-222 (miR-222) has been reported to be involved in the initiation, development and metastasis of tumors, as well as conferring resistance to chemotherapeutic drugs or radiotherapy in various types of cancer. However, the role and the underlying molecular mechanism of miR-222 specifically in nasopharyngeal carcinoma (NPC) remains unclear. Thus, the biological function and underlying mechanism of in miR-222 was investigated in NPC tissue specimens and cell lines. miR-222 was upregulated in NPC tissues and malignant cell lines compared with adjacent normal samples and cell lines. miR-222 upregulation significantly increased NPC cell proliferation, colony formation and cell apoptosis. Furthermore, miR-222 upregulation conferred radioresistance. It was also confirmed that phosphatase and tensin homolog (PTEN) was a direct target for miR-222 in NPC cells. Alteration of miR-222 expression was demonstrated to regulate the phosphoinositide 3-kinase/protein kinase B pathway in NPC cells. These results suggest that miR-222 may act as an oncomir in NPC by targeting PTEN, and has potential as a therapeutic target in NPC.
\end{abstract}

\section{Introduction}

Nasopharyngeal carcinoma (NPC) is a malignant cancer derived from epithelial cells located in the nasopharynx (1). Radiotherapy is one of the most common treatment options for patients with NPC (2). However, due to distant metastases and local recurrence in some patients with NPC (3), radioresistance can be a serious obstacle to therapy success. Therefore, there is an urgent medical need to understand the molecular mechanisms of NPC progression and radioresistance, to

Correspondence to: Dr Chao Du, Department of Neurosurgery, China-Japan Union Hospital of Jilin University, 126 Xiantai Street, Changchun, Jilin 130033, P.R. China

E-mail: duchaos25@sina.com

Key words: nasopharyngeal carcinoma, microRNA-222, phosphatase and tensin homolog, radioresistance develop novel diagnostic and therapeutic strategies that can potentially enhance tumor cell radiosensitivity.

MicroRNAs (miRNAs) are a class of endogenous single stranded non-coding RNAs of 18-25 nucleotides in length that regulate gene expression by binding to the 3 'untranslated regions (3'UTRs) of target mRNAs $(4,5)$. Accumulating evidence has demonstrated that miRNAs regulate numerous physiological processes, including cell proliferation, cell cycle stage, apoptosis, migration, invasion and differentiation $(6,7)$. Growing evidence supports the critical role of miRNAs in the progression of human cancers, where they function as either oncogenic miRNAs (oncomirs) or tumor suppressors through the regulation of cellular proliferation, differentiation and apoptosis (8-10). A number of studies have demonstrated that miRNAs are involved in cellular ionizing radiation (IR) responses through cell cycle regulation and the apoptosis signal pathway in several types of cancer, including NPC (11-13).

miRNA-222 (miR-222) has been demonstrated to function as an oncomir in various types of human cancer, with effects on cell growth, oncogenesis, invasion, migration and drug resistance of tumor cells (14-18). A recent report revealed that miR-222 confers radioresistance in glioblastoma cells through the activation of the protein kinase B (AKT) signaling pathway, independent of phosphatase and tensin homolog (PTEN) status (17). However, the detailed function of miR-222 in NPC remains unclear. The present study investigated the role of miR-222 in NPC carcinogenesis, particularly in NPC radioresistance.

\section{Materials and methods}

Cell culture and tissue samples. The three human NPC cell lines (HONE-1, C666-1 and TWO3) and the NP69 human immortalized nasopharyngeal epithelial cell line were obtained from The Cell Bank of Type Culture Collection of Chinese Academy of Science (Shanghai, China) were cultured in Dulbecco's modified Eagle's medium (DMEM; Gibco; Thermo Fisher Scientific, Inc., Waltham, MA, USA) containing $10 \%$ fetal bovine serum (Thermo Fisher Scientific, Inc.), $100 \mathrm{U} / \mathrm{ml}$ penicillin (Sigma Aldrich; Merck KGaA, Darmstadt, Germany) and $100 \mathrm{mg} / \mathrm{ml}$ streptomycin at $37^{\circ} \mathrm{C}$ in a humidified atmosphere of $5 \% \mathrm{CO}_{2}$. 
Human NPC and adjacent normal tissue samples $(n=30$ each) were harvested at The Tumor Hospital of Jilin Province (Changchun, China) between July 2014 and July 2015. All tissue samples were immediately snap-frozen in liquid nitrogen following surgery, and stored in liquid nitrogen until use. The study was approved by the Ethic Committee of the Tumor Hospital of Jilin Province (Changchun, China) and written informed consent was obtained from every patient

Cell transfection. miR-222 mimic, 5'-AGCUACAUCGGC UACUGGGUUU-3' and the corresponding negative control, miR-NC, UUCUCCGAACGUGUGUCACGUTT, miR-222 inhibitors, anti-miR-222, 5'-AGCUACAUCUGGCUACUG GGU-3' and the corresponding miRNA negative control, anti-miR-NC, 5'-UCUACUCUUUCUAGGAGGUUGUGA-3', were obtained from Qiagen Sciences, Inc. (Frederick, MD, USA). A PTEN overexpression plasmid was obtained from Shanghai GenePharma Co., Ltd. (Shanghai, China). Plasmids (100 ng) and miRNAs (100 nM) were transiently transfected into C666-1 cells using Lipofectamine 3000 Reagent (Invitrogen; Thermo Fisher Scientific, Inc.) according to the manufacturer's protocol. Transfected cells were cultured for 1-3 days until subsequent analysis.

Reverse transcription-quantitative polymerase chain reaction $(R T-q P C R)$. Total RNA of cell lines was extracted with TRIzol Reagent (Invitrogen; Thermo Fisher Scientific, Inc.) and the purity and concentration of the RNA was determined by a dual-beam ultraviolet spectrophotometer (Eppendorf, Hamburg, Germany). The total RNA was then reverse transcribed into cDNA using a Universal cDNA synthesis kit (Exiqon, Inc., Woburn, MA, USA) according to the manufacturer's protocol. cDNA was amplified and quantified using the Strotatagene Mx3005P real-time PCR System (Agilent Technologies, Inc., Santa Clara, CA, USA) with the Taqman Universal PCR Master Mix (Applied Biosystems; Thermo Fisher Scientific, Inc.). Specific primer sequences were: Mature miR-222 forward, ACACTCCAGCTGGGAGCT ACATCTGGCTACTG and reverse, CTCAACTGGTGTCGT GGA) and U6 (control) forward, CTCGCTTCGGCAGCA CA and reverse, AACGCTTCACGAATTTGCGT (Applied Biosystems; Thermo Fisher Scientific, Inc. PTEN, forward, TTGTGGTCTGCCAGCTAAA and reverse, CGCTCTATA CTGCAAATGCT and GAPDH forward, GCACCGTCAAGG CTGAGAAC and reverse-TGGTGAAGACGCCAGTGGA. Primers were used in this study as described previously (17). qPCR was performed in triplicate consisting of 40 cycles of a denaturation step at $95^{\circ} \mathrm{C}$ for $10 \mathrm{sec}$, annealing at $58^{\circ} \mathrm{C}$ for $30 \mathrm{sec}$ and extension at $72^{\circ} \mathrm{C}$ for $40 \mathrm{sec}$ following a cycle of a pre-denaturation step at $95^{\circ} \mathrm{C}$ for $40 \mathrm{sec}$. U6 and GAPDH were used as endogenous controls for the detection of miR-222 and PTEN, respectively. For data analysis, the $2^{-\Delta \Delta C q}$ method (19) was used to calculate fold change using the Rotor-Gene 6000 Series Software 1.7 (Qiagen Sciences, Inc.).

Cell viability assay. For the cell viability assay, transfected cells were seeded in 96-well plates at a density of $2 \times 10^{3}$ cells/well. Cells were continually cultured for $72 \mathrm{~h}$ prior to the addition of $10 \mu \mathrm{l} 0.5 \mathrm{mg} / \mathrm{ml} \mathrm{MTT}$ to each well. Cells were incubated for $4 \mathrm{~h}$ at $37^{\circ} \mathrm{C}$. The medium was subsequently removed and
$100 \mu \mathrm{l}$ dimethyl sulfoxide (Sigma-Aldrich; Merck KGaA) was added to each well. Following $20 \mathrm{~min}$ of agitation, absorbance was detected at $490 \mathrm{~nm}$ with an iMark Microplate Absorbance Reader (Bio-Rad Laboratories, Inc., Hercules, CA, USA).

Colony formation assay. Transfected cells $\left(1 \times 10^{3}\right.$ per well) were seeded in 6-well plates and cultured for 10 days. Colonies were washed with phosphate-buffered saline $(\mathrm{pH}, 7.2)$ and subsequently fixed with $4 \%$ paraformaldehyde (Sigma-Aldrich; Merck $\mathrm{KGaA}$ ) at room temperature for $10 \mathrm{~min}$ and stained with $1 \%$ crystal violet (Sigma-Aldrich; Merck KGaA) at room temperature for $5 \mathrm{~min}$. Colony numbers were counted under an IX71 inverted light microscope (Olympus Corporation, Tokyo, Japan).

Cell apoptosis assay. Cell apoptosis was examined using flow cytometry in transfected cells. At 48 h post-transfection, cells were harvested and the apoptosis assay was performed using an Annexin V/Propidium Iodide Detection kit (Nanjing KeyGen Biotech Co., Ltd., Nanjing, China) in a FACS Calibur flow cytometer (BD Biosciences, Franklin Lakes, NJ, USA), according to the manufacturer's protocol. The apoptosis ratio was calculated using CellQuest software 3.4 (BD Biosciences).

In vitro radiosensitivity assay. Cells transfected with the miR-222 mimic were treated with $0,1,2,4,6$ and 8 Gy X-ray radiation, using a Faxitron RX-650 (Faxitron Bioptics, Lincolnshire, IL, USA) with $100 \mathrm{kVp}$. The dose was administered at a rate of $3 \mathrm{~Gy} / \mathrm{min}$ at room temperature. A colony formation assay was then performed on the irradiated cells., survival curve parameters were determined using a Kaplan-Meier plot. Additionally, cells transfected with miR-222 mimic or miR-NC were exposed to 4 Gy of X-ray radiation. Cells were harvested at $48 \mathrm{~h}$ after IR and a cell apoptosis assay was subsequently performed as described above.

Vector construction and luciferase activity assay. The 3'UTR of PTEN containing the putative miR-222 binding site was synthesized (GenePharma Co., Ltd) and inserted into the pGL3 control vector (Promega Corporation, Madison, WI, USA). Mutations in the miR-222 binding site of the PTEN 3'UTR were introduced with the QuikChange Site-Directed Mutagenesis kit (Stratagene; Aligent Technologies, Inc., Santa Clara, CA, USA) according to the manufacturer's protocol.

For the luciferase reporter assay, C666-1 cells were cultured in 96-well plates and co-transfected with $100 \mathrm{ng}$ pGL3 with wild type PTEN-3'UTR (Wt-PTEN-3'UTR) or pGL3 with mutant PTEN-3'UTR (Mut-PTEN-3'UTR). Additionally, $80 \mathrm{ng}$ luciferase co-reporter vector pRL-SV40 was added, alone or in combination with miR-NC (100 nM) or miR-222 mimic (100 nM), using Lipofectamine 2000 (Invitrogen; Thermo Fisher Scientific, Inc.) according to the manufacturer's protocol. Luciferase activity was measured using the Dual Luciferase Reporter Assay System (Promega Corporation, Madison, WI, USA) 48 h post-transfection. Firefly luciferase activity was normalized to Renilla luciferase activity for each transfected well.

Western blotting. Protein extracts were obtained from cultured cells using radioimmunoprecipitation lysis buffer (Beyotime 

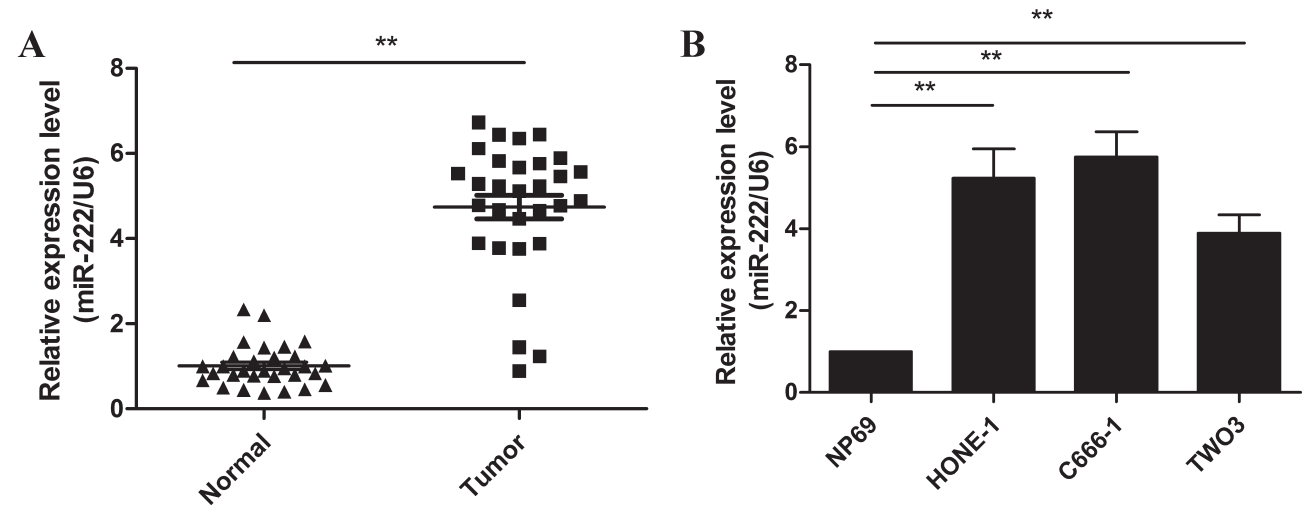

Figure 1. (A) Relative expression of miR-222 in NPC tissues ( $\mathrm{n}=30 \mathrm{NPC}$ and adjacent normal tissues). (B) Relative expression of miR-222 in three different NPC cell lines (C666-1, HONE-1 and TWO3), and a normal human nasopharyngeal epithelial cell line (NP69). Results were determined by reverse transcription-quantitative polymerase chain reaction. ${ }^{* * *} \mathrm{P}<0.01$. miR-222, microRNA-222; NPC, nasopharangeal carcinoma.

Institute of Biotechnology, Beijing, China). The total concentration of protein was measured using a bicinchoninic acid protein assay kit (Boster Biological Technology, Pleasanton, CA, USA). Protein lysates (30 $\mu \mathrm{g}$ per lane) were separated by $10 \%$ sodium dodecyl sulfate polyacrylamide gel electrophoresis (Bio-Rad Laboratories, Inc., Hercules, CA, USA), prior to transfer onto nitrocellulose membranes (Bio-Rad Laboratories, Inc.). Membranes were blocked with 5\% non-fat milk in Tris-buffered saline for $1 \mathrm{~h}$ at room temperature and incubated with the following antibodies overnight at $4^{\circ} \mathrm{C}$ : Anti-PTEN (1:1,000; sc-133197; Santa Cruz Biotechnology Inc., Dallas, TX, USA), anti-phosphoinositide 3-kinase (PI3K; 1:1,000; sc-293172; Santa Cruz), anti-AKT (1:500; sc-8312; Santa Cruz Biotechnology Inc.) and anti-phosphorylated-AKT (p-AKT; Ser473; sc-271966; 1:500; Santa Cruz Biotechnology Inc.). Anti-GAPDH (1:1,000; sc-47724; Santa Cruz Biotechnology Inc.) was used as an internal control for protein loading. The membrane was subsequently incubated with horseradish peroxidase conjugated goat anti-mouse immunoglobulin $\mathrm{G}$ ( IgG; 1:5,000; sc-516102; Santa Cruz Biotechnology Inc.) or goat anti-rabbit IgG (1:5,000; sc-2040; Santa Cruz Biotechnology Inc.) secondary antibodies for $2 \mathrm{~h}$ at room temperature. Proteins were visualized using a chemiluminescent detection system (Thermo Fisher Scientific, Inc.) and exposed on X-ray film.

Statistical analysis. All data are expressed as the mean \pm standard deviation of at least three independent experiments or samples. The statistical difference was determined using a two-tailed Student t-test or one-way analysis of variance followed by a Bonferroni post-hoc test. All data analyses were performed using SPSS 19.0 software (IBM Corp., Armonk, NY, USA). $\mathrm{P}<0.05$ was considered to indicate a statistically significant difference.

\section{Results}

miR-222 expression is upregulated in NPC tissues and cells. The expression levels of miR-222 in NPC and adjacent normal tissues was measured by RT-qPCR. As presented Fig. 1A, the level of miR-222 expression in NPC tissues was significantly higher than that of adjacent normal tissues $(\mathrm{P}<0.01)$. The expression of miR-222 in three NPC cell lines (C666-1,
HONE-1 and TWO3) and the NP69 nasopharyngeal epithelial cell line was subsequently examined. miR-222 expression was significantly upregulated in the three NPC cell lines compared with NP69 cells $(\mathrm{P}<0.05$; Fig. 1B). This data suggests that miR-222 may be involved in the initiation and progression of NPC.

miR-222 overexpression increased cell viability and colony formation, and inhibits the apoptosis of NPC cells. To examine the biological effects of miR-222 in NPC cells, C666-1 cells were transfected with miR-222 mimic or anti-miR-222 and the biological function of miR-222 in the C666-1 cells was subsequently evaluated. RT-qPCR analysis confirmed that C666-1 cells transfected miR-222 mimic had an upregulation of miR-222 expression, whereas transfection with anti-miR-222 resulted in a downregulation in miR-222 expression compared with NCs (Fig. 2A). MTT assay indicated that miR-222 mimic transfected C666-1 cells had increased cell proliferation compared with miR-NC. Conversely, a significant decrease in proliferation was observed in cells transfected with anti-miR-222 compared with anti-miR-NC (Fig. 2B). Similar results were obtained in the colony formation assay. As presented in Fig. 2C, upregulated miR-222 expression promoted colony formation, whereas downregulated miR-222 expression inhibited colony formation in C666-1 cells compared with the negative controls. Flow cytometry was subsequently used to examine the role of miR-222 in apoptosis (data not presented). It was demonstrated that downregulated miR-222 expression induced apoptosis, whereas upregulated miR-222 expression decreased cell apoptosis compared with the negative control groups (Fig. 2D). These results suggest that miR-222 functions as an oncomir in NPC cells.

miR-222 confers radioresistance in NPC cells. It is well established that certain miRNAs can regulate the radioresistance of cancer cells $(11-13,17,18)$. Thus, the effect of miR-222 on NPC cell radioresistance was investigated. C666-1 cells were transfected with miR-222 mimic prior to treatment with different doses of radiation. Colony formation assays were subsequently performed and survival curve parameters were counted. The survival rate of miR-222 mimic transfected cells significantly increased, whereas the survival rate of miR-NC transfected 


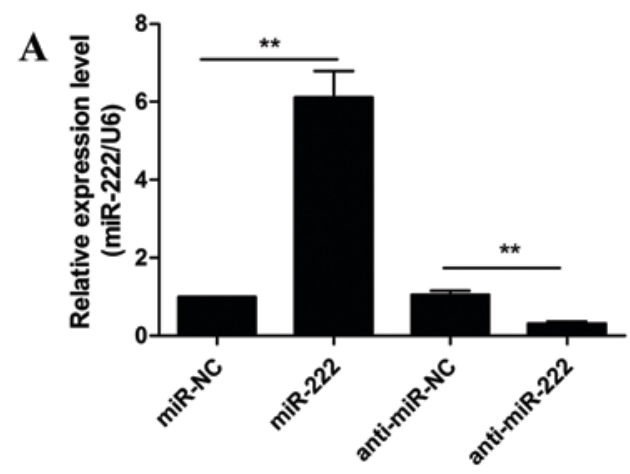

B
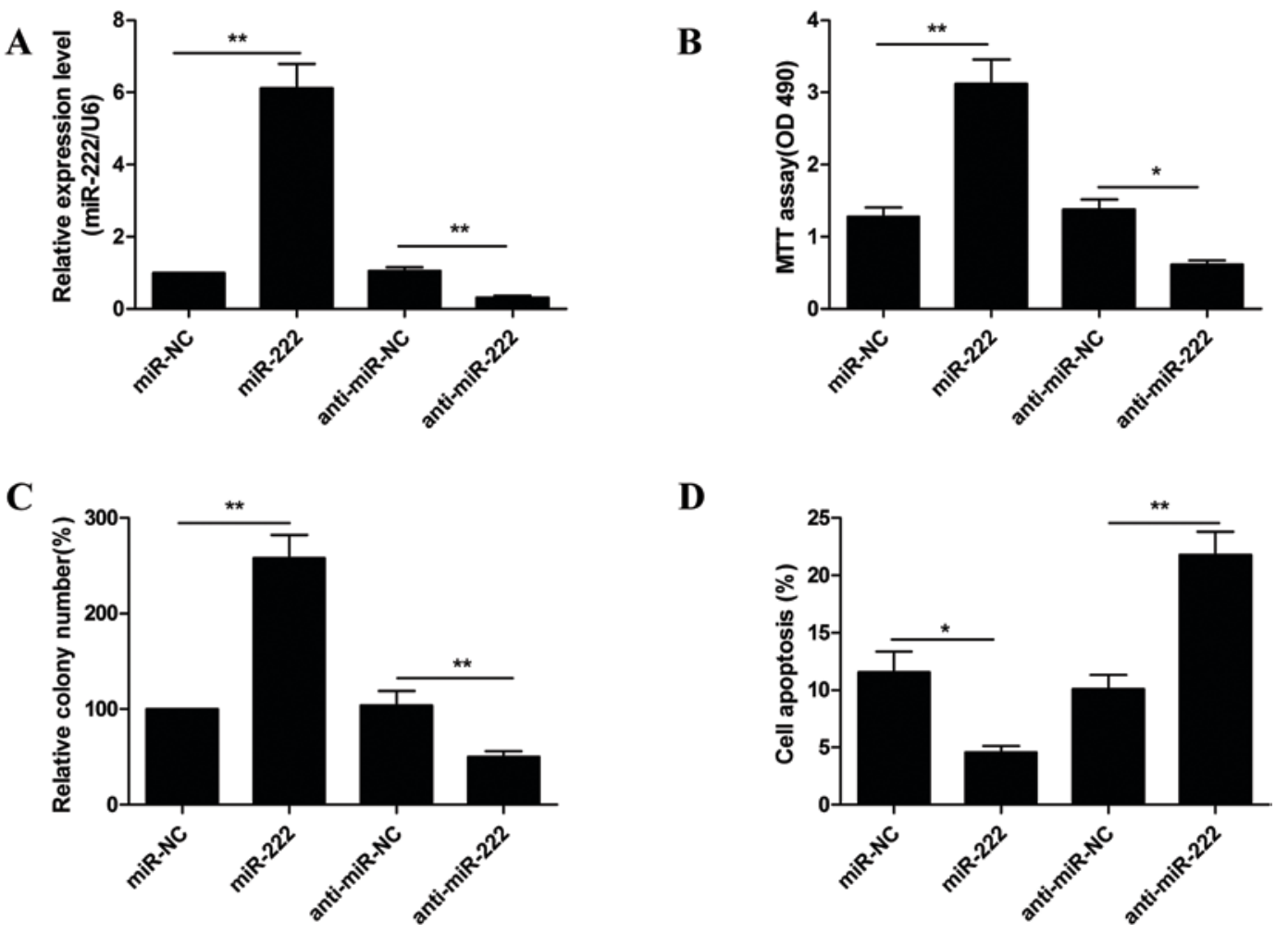

Figure 2. Effect of miR-222 on cell viability, colony formation and apoptosis of NPC cells. (A) Reverse transcription-quantitative polymerase chain reaction was performed to quantify miR-222 expression in C666-1 cells transfected with miR-222 mimic or anti-miR-222. (B) Cell viability, (C) colony formation and (D) apoptosis were determined in C666-1 cells transfected with miR-222 mimic or anti-miR-222. ${ }^{*} \mathrm{P}<0.05,{ }^{* *} \mathrm{P}<0.01$. miR-222, microRNA-222; anti-miR-222, miR-222 inhibitor. NC, negative control; OD 490, optical density $490 \mathrm{~nm}$.

A

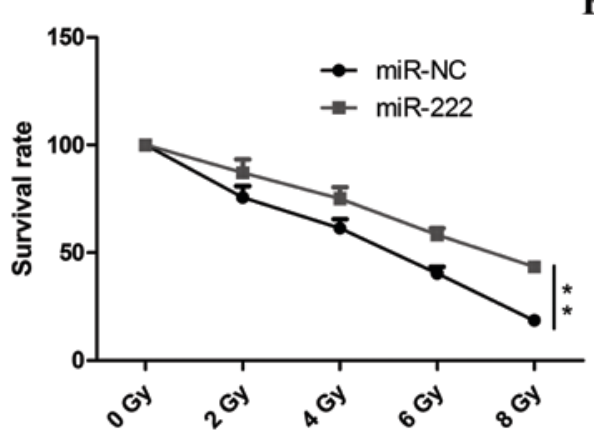

B

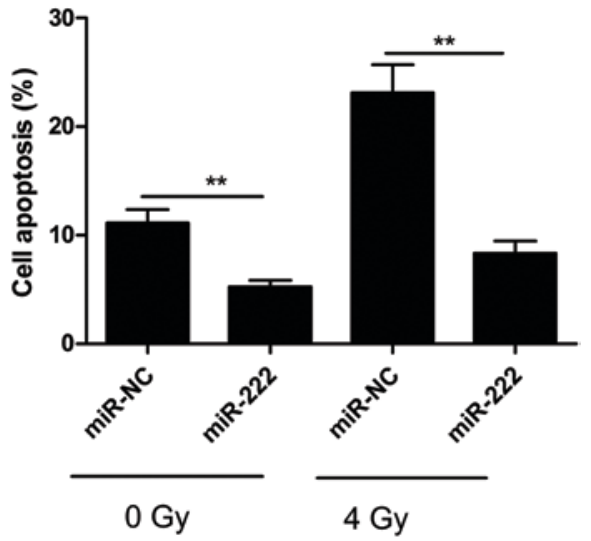

Figure 3. miR-222 confers radioresistance in NPC cells. (A) Clonogenic survival assays of C666-1 cells treated with miR-222 mimic or miR-NC followed by increasing doses of irradiation. Surviving fractions were calculated using a Kaplan-Meier plot. (B) Cell apoptosis was determined in C666-1 cells treated with miR-222 mimic or miR-NC, followed by 4 Gy of X-ray radiation. ${ }^{* *} \mathrm{P}<0.01$. miR-222, microRNA-222; miR-NC, miR-222 negative control.

cells significantly decreased (Fig. 3A). The apoptotic rate in cells overexpressing miR-222 following IR was also examined by flow cytometry (data not presented). miR-222 overexpression significantly decreased radiation-induced apoptosis in C666-1 cells at 0 Gy and 4 Gy (Fig. 3B), suggesting that miR-222 may increase radioresistance in NPC cells.

PTEN is a direct target of miR-222 in NPC cells. PTEN has been previously identified as a direct target of miR-222 in several types of cancer $(17,18)$. However, the link between miR-222 and PTEN in NPC remains unclear. To verify if PTEN is a direct target of miR-222 in NPC, a human PTEN
3'UTR fragment containing the binding sites or mutated binding sites of miR-222 (Fig. 4A) was fused to a luciferase reporter vector and co-transfected with miR-222 mimic or miR-NC into C666-1 cells. The luciferase reporter assay was subsequently performed. As presented in Fig. 4B, the relative luciferase activity was reduced following co-transfection with miR-222 mimic and Wt-PTEN-3'UTR, compared with co-transfection with miR-222 mimic and Mut-PTEN-3'UTR. To determine whether miR-222 expression affected endogenous PTEN mRNA and protein expression, miR-222 mimic, miR-NC, anti-miR-222 and anti-miR-NC were transfected into C666-1 cells for $48 \mathrm{~h}$ and were subsequently 
A

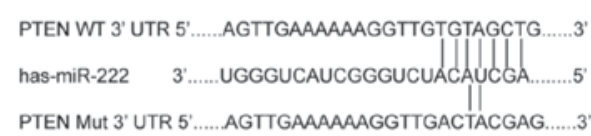

C

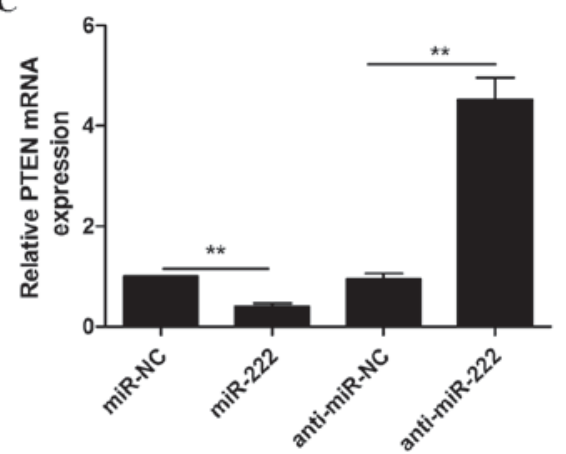

B

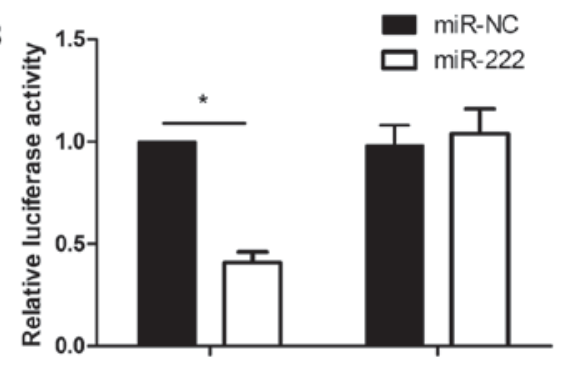

D
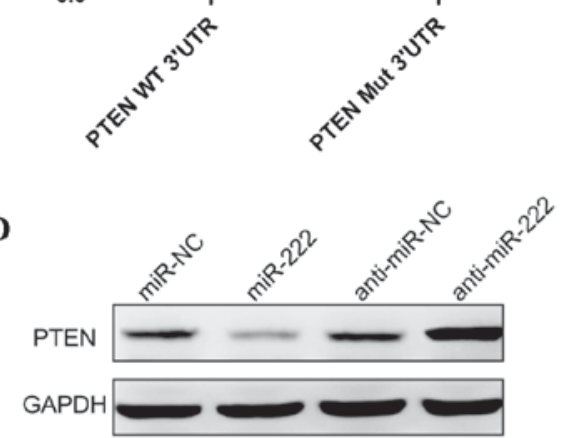

Figure 4. miR-222 targets the 3'UTR of PTEN and downregulates its expression in NPC cells. (A) The predicted binding sites for miR-222 in the 3 'UTR of PTEN and the mutations in the binding sites are presented. (B) C666-1 cells were co-transfected with PTEN WT or Mut reporter plasmids along with miR-222 mimic or miR-NC. Relative luciferase activity was measured. (C) PTEN mRNA expression and (D) protein expression was determined in C666-1 cells following transfection with miR-222 or anti-miR-222. GAPDH was used as an internal control. ${ }^{*} \mathrm{P}<0.05,{ }^{* *} \mathrm{P}<0.01$. PTEN, phosphatase and tensin homolog; WT, wild type; 3'UTR, 3'untranslated region; miR-222, microRNA-222; Mut, mutant; miR-NC, miR-222 negative control; anti-miR-222, miR-222 inhibitor.

analyzed with RT-qPCR and western blotting. The results revealed that miR-222 overexpression inhibited PTEN mRNA and protein expression in C666-1 cells, whereas reduced miR-222 expression increased PTEN mRNA and protein expression in C666-1 cells (Fig. 4C and D), suggesting that PTEN is a target of miR-222 in NPC cells.

miR-222 regulates the PI3K/AKT signaling pathway in NPC cells. PTEN has been reported to act as a negative regulator of the PI3K/AKT pathway, via the dephosphorylation of phosphatidylinositol $(3,4,5)$-triphosphate $(20)$. To investigate if miR-222 could regulate the PI3K/AKT pathway, PI3K, AKT and p-AKT protein levels were detected by western blot in miR-222 mimic or anti-miR-222 transfected C666-1 cells. PI3K and p-AKT protein expression levels were increased in miR-222 mimic transfected cells, and decreased in anti-miR-222-transfected cells compared with the negative controls (Fig. 5). AKT protein expression was unchanged among the groups (Fig. 5). These findings suggest that the promotion of cell proliferation, migration and invasion by miR-222 may be mediated via the activation of the PI3K/AKT signaling pathway.

\section{Discussion}

Recent studies have demonstrated the involvement of several miRNAs in NPC initiation and development, through the regulation of target gene expression (11-13,21). The present study revealed that miR-222 expression was increased in clinical NPC tissues and cell lines, compared to adjacent normal tissues and cell lines. miR-222 was demonstrated to be involved in NPC progression through the regulation of proliferation, colony formation and cell apoptosis. Furthermore, miR-222 was observed to increase radioresistance in NPC cells. PTEN

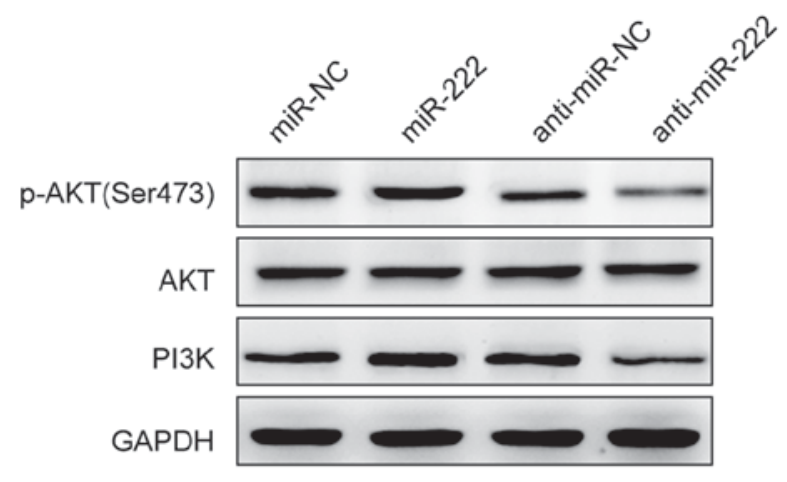

Figure 5. miR-222 regulates the PI3K/AKT signaling pathway. PI3K, AKT and p-AKT protein expression was determined in C666-1 cells after transfection with miR-222 mimic or anti-miR-222. GAPDH was used as an internal control. miR, microRNA; NC, negative control; anti-miR-222, miR-222 inhibitor; AKT, protein kinase B; p-AKT, phosphorylated-AKT; PI3K, phosphoinositide 3-kinase.

was verified as a direct, functional target of miR-222 in NPC cells. These findings contribute to the understanding of NPC development and radioresistance, and suggest miR-222 as a potential target in NPC therapy.

As an oncomir, miR-222 has been widely reported to be upregulated in numerous types of human cancer, including non-small cell lung cancer (22), bladder cancer (23), breast cancer (24), glioblastoma (25) and hepatocellular carcinoma (26). Accumulating evidence suggests that miR-222 contributes to tumor development, progression, metastasis, and may be an effective biomarker or therapy target $(15-18,23-26)$. Previous studies have demonstrated that miR-222 is involved in the radioresistance of glioblastoma and gastric cancer cells $(17,18)$. However, the role of miR-222 in NPC remains elusive. In the present study, miR-222 expression was upregulated in NPC 
tissues and cell lines, and miR-222 overexpression promoted tumor growth and the radioresistance of NPC cells, suggesting that miR-222 functions as an oncomir in NPC cells.

Located on chromosome 10q23.3, PTEN is downregulated in numerous types of human cancer and functions as a tumor suppressor (27). Recent studies have revealed that PTEN expression is downregulated in NPC tissues, and that normal PTEN expression may suppress NPC development $(28,29)$. It has been reported that PTEN can regulate radioresistance in cancer cells $(17,18)$ and the PTEN/PI3K/AKT pathway $(30)$. PTEN has previously been identified as a direct target gene of miR-222 in glioblastoma cells and gastric cancer cells $(17,18)$. Consistent with these results, the present study identified PTEN as a potential target of miR-222 in NPC cells. miR-222 was also demonstrated to regulate the PI3K/AKT pathway. These results suggest that miR-222 can promote tumor growth and confer radioresistance in NPC cells by directly targeting PTEN and thus indirectly regulating the PI3K/AKT signaling pathway.

In conclusion, the present study provides evidence that miR-222 expression is upregulated in NPC tissues and cell lines, and that miR-222 may promote cell proliferation and colony formation, decrease cell apoptosis and confer radioresistance in NPC cells. Furthermore, PTEN was identified as a crucial target gene of miR-222. It was demonstrated that mir-222 inhibited PTEN expression and positively regulated the PI3K/AKT signaling pathway, suggesting that miR-222 functions as an oncomir in NPC and may be a potential therapeutic target in NPC.

\section{Acknowledgements}

The present study was supported by the Project of the Health and Family Planning Commission (2014ZC030).

\section{References}

1. Torre LA, Bray F, Siegel RL, Ferlay J, Lortet-Tieulent J and Jemal A Global cancer statistics, 2012. CA Cancer J Clin 65: 87-108, 2015.

2. Lai SZ, Li WF, Chen L, Luo W, Chen YY, Liu LZ, Sun Y, Lin AH, Liu MZ and Ma J: How does intensity-modulated radiotherapy versus conventional two-dimensional radiotherapy influence the treatment results in nasopharyngeal carcinoma patients? Int $\mathbf{J}$ Radiat Oncol Biol Phys 80: 661-668, 2011.

3. Xiao WW, Huang SM, Han F, Wu SX, Lu LX, Lin CG, Deng XW, Lu TX, Cui NJ and Zhao C: Local control, survival, and late toxicities of locally advanced nasopharyngeal carcinoma treated by simultaneous modulated accelerated radiotherapy combined with cisplatin concurrent chemotherapy: Long-term results of a phase 2 study. Cancer 117: 1874-1883, 2011.

4. Brennecke J and Cohen SM: Towards a complete description of the microRNA complement of animal genomes. Genome Biol 4: $228,2003$.

5. Ambros V: The functions of animal microRNAs. Nature 431: 350-355, 2004

6. Bartel DP: MicroRNAs: Genomics, biogenesis, mechanism, and function. Cell 116: 281-297, 2004

7. Carthew RW and Sontheimer EJ: Origins and Mechanisms of miRNAs and siRNAs. Cell 136: 642-655, 2009.

8. Esquela-Kerscher A and Slack FJ: Oncomirs-microRNAs with a role in cancer. Nat Rev Cancer 6: 259-269, 2006.

9. Lu J, Getz G, Miska EA, Alvarez-Saavedra E, Lamb J, Peck D, Sweet-Cordero A, Ebert BL, Mak RH, Ferrando AA, et al: MicroRNA expression profiles classify human cancers. Nature 435: 834-838, 2005.

10. Volinia S, Calin GA, Liu CG, Ambs S, Cimmino A, Petrocca F, Visone R, Iorio M, Roldo C, Ferracin M, et al: A microRNA expression signature of human solid tumors defines cancer gene targets. Proc Natl Acad Sci USA 103: 2257-2261, 2006.
11. Kang M, Xiao J, Wang J, Zhou P, Wei T, Zhao T and Wang R: MiR-24 enhances radiosensitivity in nasopharyngeal carcinoma by targeting SP1. Cancer Med 5: 1163-1173, 2016.

12. Lin T, Zhou F,Zhou H, Pan X, Sun Z and Peng G: MicroRNA-378g enhanced radiosensitivity of NPC cells partially by targeting protein tyrosine phosphatase SHP-1. Int J Radiat Biol 91: 859-866, 2015.

13. Zhao L, Tang M, Hu Z, Yan B, Pi W, Li Z, Zhang J, Zhang L, Jiang W, Li G, et al: miR-504 mediated down-regulation of nuclear respiratory factor 1 leads to radio-resistance in nasopharyngeal carcinoma. Oncotarget 6: 15995-16018, 2015.

14. Miller TE, Ghoshal K, Ramaswamy B, Roy S, Datta J, Shapiro CL, Jacob S and Majumder S: MicroRNA-221/222 confers tamoxifen resistance in breast cancer by targeting p27Kip1. J Biol Chem 283: 29897-29903, 2008.

15. Rao X, Di Leva G, Li M, Fang F, Devlin C, Hartman-Frey C, Burow ME, Ivan $M$, Croce CM and Nephew KP: MicroRNA-221/222 confers breast cancer fulvestrant resistance by regulating multiple signaling pathways. Oncogene 30: 1082-1097, 2011.

16. Lee JC, Zhao JT, Clifton-Bligh RJ, Gill A, Gundara JS, Ip JC, Glover A, Sywak MS, Delbridge LW, Robinson BG and Sidhu SB: MicroRNA-222 and microRNA-146b are tissue and circulating biomarkers of recurrent papillary thyroid cancer. Cancer 119: 4358-4365, 2013.

17. Li W, Guo F, Wang P, Hong S and Zhang C: miR-221/222 confers radioresistance in glioblastoma cells through activating AKT independent of PTEN status. Curr Mol Med 14: 185-195, 2014.

18. Chun-Zhi Z, Lei H, An-Ling Z, Yan-Chao F, Xiao Y, Guang-Xiu W, Zhi-Fan J, Pei-Yu P, Qing-Yu Z and Chun-Sheng K: MicroRNA-221 and microRNA-222 regulate gastric carcinoma cell proliferation and radioresistance by targeting PTEN. BMC Cancer 10: 367, 2010.

19. Livak KJ and Schmittgen TD: Analysis of relative gene expression data using real-time quantitative PCR and the 2(-Delta Delta C(T)) method. Methods 25: 402-408, 2001.

20. Yamada KM and Araki M: Tumor suppressor PTEN: Modulator of cell signaling, growth, migration and apoptosis. J Cell Sci 114: 2375-2382, 2001

21. Wang Y, Guo Z, Shu Y, Zhou H, Wang H and Zhang W: BART miRNAs: An unimaginable force in the development of nasopharyngeal carcinoma. Eur J Cancer Prev 26: 144-150, 2017.

22. Yamashita R, Sato M, Kakumu T, Hase T, Yogo N, Maruyama E, Sekido Y, Kondo M and Hasegawa Y: Growth inhibitory effects of miR-221 and miR-222 in non-small cell lung cancer cells. Cancer Med 4: 551-564, 2015.

23. Zhang DQ, Zhou CK, Jiang XW, Chen J and Shi BK: Increased expression of miR-222 is associated with poor prognosis in bladder cancer. World J Surg Oncol 12: 241, 2014.

24. Hwang MS, Yu N, Stinson SY, Yue P, Newman RJ, Allan BB and Dornan D: miR-221/222 targets adiponectin receptor 1 to promote the epithelial-to-mesenchymal transition in breast cancer. PLoS One 8: e66502, 2013.

25. Quintavalle C, Garofalo M, Zanca C, Romano G, Iaboni M, del Basso De Caro M, Martinez-Montero JC, Incoronato M, Nuovo G, Croce CM and Condorelli G: miR-221/222 overexpession in human glioblastoma increases invasiveness by targeting the protein phosphate PTPu. Oncogene 31: 858-868, 2012.

26. Wong QW, Ching AK, Chan AW, Choy KW, To KF, Lai PB and Wong N: MiR-222 overexpression confers cell migratory advantages in hepatocellular carcinoma through enhancing AKT signaling. Clin Cancer Res 16: 867-875, 2010.

27. Zhang S and Yu D: PI(3)king apart PTEN's role in cancer. Clin Cancer Res 16: 4325-4330, 2010.

28. Cai LM, Lyu XM, Luo WR, Cui XF, Ye YF, Yuan CC, Peng QX, Wu DH, Liu TF, Wang E, et al: EBV-miR-BART7-3p promotes the EMT and metastasis of nasopharyngeal carcinoma cells by suppressing the tumor suppressor PTEN. Oncogene 34: 2156-2166, 2015.

29. Zhou XM, Sun R, Luo DH, Sun J, Zhang MY, Wang MH, Yang Y, Wang HY and Mai SJ: Upregulated TRIM29 promotes proliferation and metastasis of nasopharyngeal carcinoma via PTEN/AKT/mTOR signal pathway. Oncotarget 7: 13634-13650, 2016.

30. Hafsi S, Pezzino FM, Candido S, Ligresti G, Spandidos DA, Soua Z, McCubrey JA, Travali S and Libra M: Gene alterations in the PI3K/PTEN/AKT pathway as a mechanism of drug-resistance (review). Int J Oncol 40: 639-644, 2012. 